\title{
The Study of Viral RNA Diversity in Bird Samples Using De Novo Designed Multiplex Genus-Specific Primer Panels
}

\author{
Andrey A. Ayginin $\mathbb{D}^{1,}$, Ekaterina V. Pimkina, $^{1}$ \\ Alina D. Matsvay $\mathbb{D}^{1,2}$ Anna S. Speranskaya ${ }^{1}{ }^{1,3}$ Marina V. Safonova, ${ }^{1}$ \\ Ekaterina A. Blinova, ${ }^{1}$ Ilya V. Artyushin $\mathbb{D}^{3},{ }^{3}$ Vladimir G. Dedkov $\mathbb{D}^{1,4}$ \\ German A. Shipulin, ${ }^{1}$ and Kamil Khafizov $\mathbb{D D}^{1,2}$ \\ ${ }^{1}$ Central Research Institute of Epidemiology, Moscow 111123, Russia \\ ${ }^{2}$ Moscow Institute of Physics and Technology, Dolgoprudny 141700, Russia \\ ${ }^{3}$ Lomonosov Moscow State University, Moscow 119991, Russia \\ ${ }^{4}$ Saint-Petersburg Pasteur Institute, Saint Petersburg 197101, Russia
}

Correspondence should be addressed to Kamil Khafizov; kkhafizov@gmail.com

Received 11 May 2018; Revised 4 July 2018; Accepted 24 July 2018; Published 12 August 2018

Academic Editor: Gary S. Hayward

Copyright (C) 2018 Andrey A. Ayginin et al. This is an open access article distributed under the Creative Commons Attribution License, which permits unrestricted use, distribution, and reproduction in any medium, provided the original work is properly cited.

\begin{abstract}
Advances in the next generation sequencing (NGS) technologies have significantly increased our ability to detect new viral pathogens and systematically determine the spectrum of viruses prevalent in various biological samples. In addition, this approach has also helped in establishing the associations of viromes with many diseases. However, unlike the metagenomic studies using $16 S$ rRNA for the detection of bacteria, it is impossible to create universal oligonucleotides to target all known and novel viruses, owing to their genomic diversity and variability. On the other hand, sequencing the entire genome is still expensive and has relatively low sensitivity for such applications. The existing approaches for the design of oligonucleotides for targeted enrichment are usually involved in the development of primers for the PCR-based detection of particular viral species or genera, but not for families or higher taxonomic orders. In this study, we have developed a computational pipeline for designing the oligonucleotides capable of covering a significant number of known viruses within various taxonomic orders, as well as their novel variants. We have subsequently designed a genus-specific oligonucleotide panel for targeted enrichment of viral nucleic acids in biological material and demonstrated the possibility of its application for virus detection in bird samples. We have tested our panel using a number of collected samples and have observed superior efficiency in the detection and identification of viral pathogens. Since a reliable, bioinformatics-based analytical method for the rapid identification of the sequences was crucial, an NGS-based data analysis module was developed in this study, and its functionality in the detection of novel viruses and analysis of virome diversity was demonstrated.
\end{abstract}

\section{Introduction}

An increase in globalization, climate change, and interaction with livestock animals has resulted in the emergence of novel viral pathogens or zoonoses [1], which pose a serious health problem for birds and animals and ultimately for humans. The natural reservoirs of pathogens, such as birds, bats, rodents, and bloodsucking arthropods play a significant role in the sustenance and transmission of zoonotic infections. Migratory birds warrant special attention, as their rich diversity and migratory behavior contribute to the spread of infections to considerable distances. Such migrations are strongly associated with the emergence of the epidemic and enzootic outbreaks as well as the formation and activation of natural sources of viral infections. Wild birds are widely acknowledged to be reservoirs and transmitters of pathogens responsible for emerging infectious diseases such as severe acute respiratory syndrome virus (SARSV), avian influenza virus A (H10N7), and West Nile virus (WNV), to domestic animals and humans $[2,3]$. The rich biodiversity of the 
wild bird population may increase the risk of spread of pathogens to domesticated poultry. Understanding the viral diversity is critical for predicting future risks of transmission or possible outbreaks of viral diseases. However, identifying and monitoring the transmission of novel viruses are one of the vital requisites for responding to outbreaks. The asymptomatic carriage of viruses, which could be attributed to certain characteristics of bird metabolism and the adaptive capabilities of their immune system, provides ideal conditions for coevolution, leading to the emergence of mutant and recombinant strains of viruses. Hence, the majority of widely used molecular diagnostic methods, such as those employing polymerase chain reaction (PCR), are not sufficiently suitable for the identification of a wide variety of viruses, as the techniques are usually designed for the detection of highly conserved regions in the genomes, which limits the search to a restricted group of viral agents and prevents the identification of new viruses or viral variants. In addition, the Sanger sequencing technique, which has been a standard diagnostic tool for the detection and identification of various pathogens, provides limited sequence information at a higher cost per nucleotide base and can only be used to identify pathogens with a high titer. Moreover, a preliminary evidence of the presence of the certain viruses is required for performing the PCR, in the absence of which the process of pathogen identification could take a significant amount of time, which can be a major obstacle in the prevention and control of the infection.

DNA barcoding is a method which uses a short part of organism's genome (so-called barcode) to identify whether it belongs to a particular family, genera, or even species, by using extensive parallel sequencing technologies (or more commonly NGS [Next Generation Sequencing]). This method was initially developed for studying bacterial communities (e.g., studies on gut microbiota), but today it is widely employed for various tasks, including detection of food adulteration [4], the study of the diets of marine communities [5], and biofuel analysis [6]. Unlike other taxa, viruses lack a universally shared phylogenetic marker (such as $16 \mathrm{~S}$ for bacteria, Cytochrome $C$ oxidase for birds and mammals, $r b c L$ and $m a t K$ for plants, and Internal Transcribed Spacer (ITS) for fungi or plants), which makes it impossible to design a universal primer pair to amplify and differentiate diverse viral sequences. Furthermore, the viral taxonomy gives a better indication of the signs of diseases caused, rather than genetic similarity. This fact complicates the barcode (a short, standardized nucleotide sequence of an organism's DNA) selection, even for one genus (for example, mammarenavirus can be serologically, phylogenetically, and geographically divided into two major complexes: the Old World complex prevalent in Africa, Europe, and Asia, and the New World complex found in North and South America [7]), let alone higher taxa. However, metagenomics still allows the detection of different viral pathogens using shotgun sequencing [8]. Despite the constant reduction in the cost of DNA sequencing, this approach is still considerably expensive and is not feasible for screening a large number of samples. Also, metagenomic NGS data-sets are usually predominantly composed of host-derived sequences with only a minor fraction of pathogen sequences. Often, even an approximate fraction of pathogen content is initially unknown, making it practically impossible to estimate how many sequencing reads are needed per sample, in order to detect the pathogen in the final sequencing data file. Another common problem for most NGS-based tests is that complex multistep workflows may pose challenges in the reproducibility of results. Recently, Briese et al. [9] had developed a virome capture sequencing platform for vertebrate viruses (VirCapSeq-VERT), which consisted of $\sim 2$ million biotinylated DNA-probes for target enrichment of viral nucleic acids to increase the sensitivity of sequence-based detection and characterization of viruses. The described method allowed the identification (and possibly even sequencing the whole genome assembly of detected viruses) of a large number of viruses including the novel ones. However, the overall cost of sequencing per sample remained considerably high.

Other methods of enrichment are based on the targeted amplification of cDNA region using genus-specific primer pairs in PCR. This approach has been known for a long time [10] and has been used successfully by various researchers, both in the studies of the representatives of individual viral genera and in the analyses of the diversity of viruses in different types of biological material [11-13]. Till recently, a large number of such primers have been described [14-16]. However, most of them were designed for the detection of certain species of viruses. Moreover, it was impossible to use them in multiplex reactions due to primer-specific annealing temperatures, nonspecific amplification, and potential selfcomplementarity. Thus, in order to analyze one sample, it is necessary to carry out a number of PCR experiments corresponding to the number of primer pairs. The effectiveness of the study could be significantly increased by combining genus-specific PCR and NGS. In this approach, the products of different PCR assays for each sample would be pooled in one tube, purified, eluted in a minimal volume, and prepared for NGS [17]. However, this approach does not exempt the requirement of many PCRs per sample, which is a problem when a large number of samples are studied simultaneously. In addition, there are restrictions on the use of different protocols for the library preparation, particularly when the protocol includes the emulsion PCR stage, which strictly requires PCR products of the same length.

In this study, we have introduced a method for designing oligonucleotide panels for targeted enrichment of viral nucleic acids, where the main objective is to use a minimum number of primer oligonucleotides to cover the maximum number of diverse viral taxa within a single PCR reaction. We have applied this approach to design genus-specific primer pairs for targeted enrichment of cDNA from zoonotic RNA viruses and have evaluated it using several samples from birds. We have also demonstrated a considerable increase in the viral genome coverage.

\section{Materials and Methods}

2.1. Design of the Genus-Specific Primers Panel. This section contains the technical details of the algorithm for the design of genus-specific primer panels. To enable us to process 
the enrichment PCR reaction in a single tube, a number of restriction parameters were applied to the positions and structures of oligonucleotides. The availability of validated reference viral nucleic acid sequences is crucial for efficient usage of the algorithm. Although several viral reference sequence databases are publicly available [18], the medically relevant and model organisms are largely overrepresented in most of them, and the genomic diversity of circulating strains is often underrepresented. One such source is the open-source database "The Virus Pathogen Database and Analysis Resource (ViPR)" [19], which was developed back in 2011. The most important advantage of this database is the authenticity of nucleic acid sequences, as the data are curated and managed by experts in virology and bioinformatics. This source was used to retrieve the sequences of the polymerase genes of target viral genera (or other genes in cases where the sequences of polymerase genes were not available). The sequences were filtered by length $(\geq 400 \mathrm{bp})$, quality, and intrageneric similarity and were combined to the corresponding FASTA files.

In order to create consensus sequences, nucleotide sequences within each FASTA file specific to a genus were aligned using ClustalW [20]. If a minimum of two consensus subsequences with lengths of $20 \mathrm{bp}$ and a maximum of four ambiguous positions with minor nucleotide frequency $\geq 10 \%$ were not identified, the original FASTA file was iteratively clustered using CD-HIT [21], with decreasing threshold. The clusters obtained at each step were aligned independently using ClustalW to identify consensus subsequence(s) for all the subsets, which must collectively represent at least $90 \%$ of different species within the genus. Finally, at least one aligned FASTA file was obtained for every genus.

For extracting the common subsequences from multiple sequence alignments, a "sliding window" was used within a specified range of lengths. This window "slides" from the 5'to 3 '-end of the alignment with a step of one nucleotide and identifies all subsequences fulfilling the following criteria:

(i) proportion of ambiguous positions $\left(P_{A M B}\right) \leq 20 \%$;

(ii) proportion of unique species, which share the subsequence and do not contain gaps $\left(P_{S H}\right) \geq 50 \%$;

(iii) GC content of the consensus sequence $\left(P_{G C}\right)$ within $35-65 \%$ interval;

(iv) absence of self-complementary regions;

(v) absence of formation of homodimers;

(vi) absence of formation of heterodimers with previously selected oligonucleotides.

An amplicon length between two subsequences (primers) was then adjusted between 200 and 400 bp to make the panel compatible with the most popular sequencing platforms (for example, Illumina MiSeq or Ion S5 from Thermo Fisher Scientific). Two subsequences were considered to be a pair if they together covered at least $90 \%$ of species related to a target genus or cluster and shared over $90 \%$ of the species. The pairs were then filtered according to their annealing temperature $\left(50^{\circ} \mathrm{C} \leq \mathrm{T}_{\mathrm{a}} \leq 55^{\circ} \mathrm{C}\right)$. The selected primer pairs were then aligned with the NR database using BLAST to check for their specificity. Nonspecific candidates were eliminated. The possibility of formation of heterodimers between sequences in the pair, and between the sequences and previously selected primers, was calculated using the software Primer3 [22]. Then the parameters described above were calculated and the pairs were sorted accordingly. The "best" primer pair was selected as a "genus-specific pair."

The parameters described above were then calculated, and the pairs were sorted accordingly. The primer pair with the best fit was selected as a "genus-specific pair."

The sequences of primer pairs for the reference viral genera, designed using the developed algorithm, are presented in Tables 1 and 2.

2.2. Control Samples. The ability of the developed panel to enrich the target cDNA from zoonotic RNA viruses was assessed using high titer solutions of viral RNA (concentration ranging from $10^{5}$ to $10^{7}$ copies per $\mathrm{mL}$ ) from 24 species of viruses, belonging to 13 viral genera within 12 viral families (Table 1). Viral RNAs were sourced from the collection stored at the Central Research Institute for Epidemiology, Moscow, Russia. All viral RNAs were stored at $-70^{\circ} \mathrm{C}$ till further use for the study. The $\mathrm{H}_{2} \mathrm{O}$ sterile (AmpliSens, Russia) was used as a negative control in all experiments.

The control samples cDNA was obtained by reverse transcription reaction performed on $5 \mu \mathrm{L}$ of the extracted RNA using the Reverta-L RT kit (AmpliSens; total volume of the reaction mixture is $20 \mu \mathrm{L}$ ); after that $5 \mu \mathrm{L}$ of the reaction mixture containing cDNAs was further used for evaluation of the ability of the primer pair to amplify the targeted region of viruses, both in single and in multiplex PCR format.

2.3. Reaction Mixture and Amplification Mode. The PCR reaction mix $(25 \mu \mathrm{L})$ was prepared using $5 \mu \mathrm{L}$ of the cDNA template, $5 \mu \mathrm{L}$ of $\mathrm{H}_{2} \mathrm{O}$ (MilliQ, AmpliSens), $5 \mu \mathrm{L}$ of PCR mix 2 FEP/FRT, $1 \mu \mathrm{L}$ of $0.2 \mu \mathrm{M}$ of each primer (in the single-plex format) or $0.08 \mu \mathrm{M}$ of each primer (total concentration of $3.84 \mu \mathrm{M}$ in the multiplex format), $2.5 \mu \mathrm{L}$ of dNTPs $(1.76 \mathrm{mM}$; AmpliSens), and $0.5 \mu \mathrm{L}$ of TaqF polymerase (AmpliSens). The thermal cycling parameters were initial denaturation at $95^{\circ} \mathrm{C}$ for $15 \mathrm{~min}$, followed by 45 cycles of $95^{\circ} \mathrm{C}$ for $15 \mathrm{~s}, 50^{\circ} \mathrm{C}$ for 120 $\mathrm{s}, 72^{\circ} \mathrm{C}$ for $30 \mathrm{~s}$, and final extension at $72^{\circ} \mathrm{C}$ for $7 \mathrm{~min}$.

2.4. Specificity of the Developed Panel. PCR products of appropriate lengths were resolved by electrophoresis on $1.5 \%$ agarose gel containing ethidium bromide. The amplicons were purified using a QIAquick PCR Purification Kit (Qiagen), following the manufacturer's instructions. The purified PCR products were then sequenced using ABI Prism 3500 sequencer (Applied Biosystems) to confirm the specificity of the reactions in a single-plex format.

2.5. Sample Collection. Bird samples (cloacal swabs and/or feces) were collected from the Enisei Ecological Station, Mirnoe (Russian Federation). The samples were collected from birds captured using mist-net for routine ornithological examination or from droppings left on the ground by geese at their stop-over sites. To prevent contamination, separate 
TABLE 1: Viral families and genera covered by the primer panel and control samples of viral RNA used.

\begin{tabular}{|c|c|c|c|c|}
\hline Primer set & Family & Genus & Control sample RNA & Acronym \\
\hline 1 & \multirow{3}{*}{ Filoviridae } & Ebolavirus & Zaire ebolavirus & ZEBOV \\
\hline 2 & & Marburgvirus & Marburg virus & MARV \\
\hline 3 & & Cuevavirus & N/A & \\
\hline 4 & Arenoviridae & Mammarenavirus & Lassa virus & LASV \\
\hline 5 & \multirow{2}{*}{ Rhabdoviridae } & Lyssavirus & Rabies virus & RABV \\
\hline 6 & & Ledantevirus & $\mathrm{N} / \mathrm{A}$ & \\
\hline 7 & \multirow{3}{*}{ Coronaviridae } & Alphacoronavirus & $\mathrm{N} / \mathrm{A}$ & \\
\hline 8 & & Betacoronavirus & $\begin{array}{l}\text { Middle East respiratory } \\
\text { syndrome coronavirus }\end{array}$ & MERS CoV \\
\hline 9 & & Gammacoronavirus & N/A & \\
\hline 10 & \multirow{3}{*}{ Reoviridae } & Orbivirus & Kemerovo virus & KEMV \\
\hline 11 & & Orthoreovirus & N/A & \\
\hline 12 & & Seadornavirus & N/A & \\
\hline 13 & Paramyxoviridae & Henipavirus & $\mathrm{N} / \mathrm{A}$ & \\
\hline 14 & Phenuiviridae & Phlebovirus & Uukuniemi virus N/A & \\
\hline 15 & Hantaviridae & Hantavirus & Dobrava virus & DOBV \\
\hline 16 & Nairoviridae & Orthonairovirus & $\begin{array}{l}\text { Crimean-Congo virus } \\
\text { hemorrhagic fever virus, } \\
\text { Paramushir virus }\end{array}$ & CCHFV, PRMV \\
\hline 17 & Peribunyaviridae & Orthobunyavirus & Inkoo virus & INKV \\
\hline 18 & Orthomyxoviridae & Thogotovirus & N/A & \\
\hline 19 & \multirow{2}{*}{ Picornaviridae } & Cardiovirus & N/A & \\
\hline 20 & & Parechovirus & N/A & \\
\hline 21 & Flaviviridae & Flavivirus & $\begin{array}{l}\text { Tick-borne encephalitis } \\
\text { virus, Yellow fever virus, } \\
\text { Japanese encephalitis virus }\end{array}$ & TBEV, YFV, JEV \\
\hline 22 & Togaviridae & Alphavirus & N/A & \\
\hline
\end{tabular}

rubber gloves and sterile cotton swabs were used for collection of each sample. The samples were stored at the collection site for up to 30 days at $4-8^{\circ} \mathrm{C}$, then for four days at room temperature during transport, and finally at $2-4^{\circ} \mathrm{C}$ while processing. Samples were stored in the transport medium 0.5 $\mathrm{mL}$ tubes for swabs containing storage solution (AmpliSens).

The samples were collected from the following species of birds: Taiga Bean Goose (Anser fabalis johanseni), Siberian Thrush (Geokichla sibirica), Song Thrush (Turdus philomelos), Fieldfare (Turdus pilaris), Redwing (Turdus iliacus), Black-Throated Thrush (Turdus atrogularis), Common Tern (Sterna hirundo), Green Sandpiper (Tringa ochropus), Common Greenshank (Tringa nebularia), Red-Throated Flycatcher (Ficedula albicilla), Temminck's Stint (Calidris temminckii), and Dusky Warbler (Phylloscopus fuscatus). No birds were harmed in this study. A total of 92 bird samples were used for further virome analysis, among which 62 samples belonged to Taiga Bean Goose and 6 to Siberian Thrush.

2.6. RNA Extraction and Reverse Transcription. Total RNA was extracted from $100 \mu \mathrm{L}$ of the resuspended sample with RNeasy Lipid Tissue Mini Kit (Qiagen) using robotic workstation QIAcube (Qiagen), following the manufacturer's protocol. The cDNA was obtained by reverse transcription reaction on $10 \mu \mathrm{L}$ of the extracted RNA using a Reverta-L RT kit (AmpliSens), according to the manufacturer's instructions.

2.7. Testing the Primer Panel with Control Samples. The primer panel was tested with a reference set of samples (Table 1). The results are presented in Figure 1. In all cases, the products of the specified range of lengths were obtained.

The multiplex system was then tested with the same set of viruses and the PCR products were reamplified using the genus-specific primer pairs. In all cases, unspecific amplification was observed as well. However, the reamplification reactions with the genus-specific primers showed the presence of the target products. The multiplex system was first tested with three bat samples infected by several known viruses (sample N1: Betacoronavirus, sample N2: Betacoronavirus, sample N3: Orthoreovirus). The reamplification of the PCR products was carried out with the genus-specific primers (Figure 2). The obtained results clearly demonstrated the presence of the products with target length in all three samples. 
TABLE 2: Primers used in the panel along with their structures.

\begin{tabular}{|c|c|c|}
\hline Primer set & Primer name & Sequence 5'-3' \\
\hline \multirow{2}{*}{1} & ebola_f & GCAATGTTCAAACACTTTGTGARGC \\
\hline & ebola_r & CTTAACACCATAGCAACGGTTR \\
\hline \multirow{2}{*}{2} & marburg $f$ & TGGACGATAGGAAATCGAGCAC \\
\hline & marburg_r & TGAACTATRTTGCCTGAGTAGTGWG \\
\hline \multirow{2}{*}{3} & cueva_f & GTGCCAGAACAGTTTGAACTCA \\
\hline & cueva_r & CCGAATTCTCTGGGTAACACAA \\
\hline \multirow{2}{*}{4} & mammarena_f & CAATMCTTGAYATGGGWCARGG \\
\hline & mammarena_r & WGATTTRAACTCTGCAACAAAYCTR \\
\hline \multirow{2}{*}{5} & lyssa_f & CTKGAYTATGARAARTGGAACA \\
\hline & lyssa_r & TATGTCGGRCAYARAACCTGRT \\
\hline \multirow[b]{2}{*}{6} & ledante_f & AAYAATACATGGCCCACWCC \\
\hline & ledante_r & ARTATTCTCTMARMKCCCARGACAT \\
\hline \multirow{2}{*}{7} & alphacorona_f & GGYACHACHTCWGGTGATGS \\
\hline & alphacorona_r & GYTTACGYARRTAACCAWAAWABTC \\
\hline \multirow{2}{*}{8} & betacorona $f$ & GTGCWAAGAATAGAGCYCGCAC \\
\hline & betacorona_r & RTCACAYTTWGGRTAATCCCAACCC \\
\hline \multirow{2}{*}{9} & gammacorona_f & CCACATCTGCTAATGTTGCR \\
\hline & gammacorona_r & CAGAAATRTCWGCTACAAGACCYTG \\
\hline \multirow{3}{*}{10} & orbi_fl & TACCGCARGATMGWATGATGAT \\
\hline & orbi_f2 & TATGTTCCWCARGATCGRATGATG \\
\hline & orbi_r & TGCGCTCCAWAVCCATTCCA \\
\hline \multirow{2}{*}{11} & orthoreo_f & GTYTCGGCGCCYCAYACDYT \\
\hline & orthoreo_r & GCAGTRTGCTCAGTDGARGT \\
\hline \multirow{2}{*}{12} & seadorna_f & CCRCATGAYGTHATGGCYCC \\
\hline & seadorna_r & TCACCWGACTTAACWCCWGM \\
\hline \multirow{2}{*}{13} & henipa_f & GGTCAGARACWYTGGTGGAYGA \\
\hline & henipa_r & ARTAYGGATCACTRGCCCARTC \\
\hline \multirow{2}{*}{14} & phlebo_f & GATTYAATCTSTKSARRGCY \\
\hline & phlebo_r & YTATYWGYTCCAYCCAGTYYTC \\
\hline \multirow{2}{*}{15} & hanta_f & GCWGATGCAACWAARTGGTC \\
\hline & hanta_r & YARRTTYCCYTGYARCCART \\
\hline \multirow{2}{*}{16} & nairo_f & CCTTCTTTTSHGGYATGATGCA \\
\hline & nairo_r & GAAGTTAACACTGNCGAWGTWGCATG \\
\hline \multirow{2}{*}{17} & orthobunya_f & CWGAWGARATGATWWSTGARCCWGG \\
\hline & orthobunya_r & GCACTCCATTTWGACATRTCWG \\
\hline \multirow{2}{*}{18} & thogoto_f & ATCAARGAYMRRCTGAARAANA \\
\hline & thogoto_r & TCGATSYGMGGCTTTATDGM \\
\hline \multirow{2}{*}{19} & cardio_f & MRGGYATGGAYCCMATGGAV \\
\hline & cardio_r & AAGTTRGARTARTCYACATCRTAGA \\
\hline \multirow{2}{*}{20} & parecho_f & GGRATYAACCCATAYAARGAYTGGC \\
\hline & parecho_r & GAYCCTGATGGCATACCRCC \\
\hline \multirow{2}{*}{21} & flavi_f & CTSCTKTGTGACATMGGDGA \\
\hline & flavi_r & TACATCTCRTGYGTGGARTTBC \\
\hline 22 & $\begin{array}{l}\text { alpha_f } \\
\text { alpha_r }\end{array}$ & $\begin{array}{c}\text { ACWCTGTTTGTSAACACWGTVRTYA } \\
\text { CTYTTYARRGGGTCTGCSACHC }\end{array}$ \\
\hline
\end{tabular}

2.8. Preparation and Sequencing of Ion S5 Libraries. The targeted sequences were enriched by multiplex PCR with the designed primer pool described above (Table 2). PCR products were cleaned using carboxyl-coated magnetic particles, commercially available as Sera-Mag Speed Beads (GE Healthcare). The concentrations of the fragments were measured using Qubit dsDNA HS Assay Kit with a Qubit 2.0 fluorimeter (Invitrogen).

The preparation of the amplicon libraries involved phosphorylation of the 5 -end and incorporation of barcoded adapters, followed by amplification of the final library. For this purpose, T4 Polynucleotide Kinase and T4 DNA Ligase (both from New England Biolabs, NEB) were used according to the manufacturer's protocol with slight modification. Amplification was performed using PCR-mix 2 FEP/FRT (AmpliSens).

The two total RNA libraries for Ion S5 high-throughput sequencing were prepared from total RNA of two bird samples (B23 and B66). The first strand of cDNA was synthesized using random primers and Reverta-L RT kit (AmpliSens). The second strand of cDNA was prepared with NEBNext Ultra Second Strand Synthesis Module of Kit \#E7530 (NEB). The double stranded DNA was fragmented by Ion Shear Plus Reagent Kit (Thermo Fisher Scientific) and 


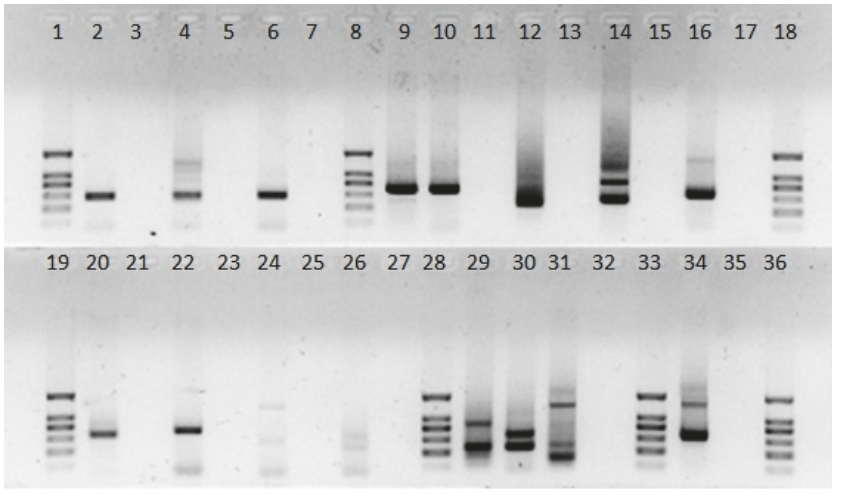

FIGURE 1: Individual primer pairs testing. $(1,8,18,19,28,33$, 36) DNA Ladder (100 bp, 200 bp, 300 bp, 400 bp, 500 bp, 800 bp); (2) ZEBOV, primers ebola_f/r; (3) negative control for primers ebola_f/r; (4) MARV, primers marburg_f/r, (5) negative control for primers marburg $f / r$; (6) LASV, primers mammarena $f / r,(7)$ negative control for primers mammarena_f/r; (9) CCHFV, primers nairo_f/r; (10) PRMV, primers nairo_f/r; (11) negative control for primers nairo_f/r; (12) INKV, primers orthobunya_f/r; (13) negative control for primers orthobunya_f/r; (14) DOBV, primers hanta_f/r; (15) negative control for primers hanta_f/r; (16) KEMV, primers orbi_fl/f2/r; (17) negative control for primers orbi_fl/f2/r; (20) MERS CoV, primers alphacorona_f/r; (21) negative control for primers alphacorona_f/r; (22) MERS (Betacoronavirus), primers betacorona_1_f/r; (23) negative control for primers betacorona_1_f/r; (24) MERS (Betacoronavirus), primers betacorona_2_f/r; (25) negative control for primers betacorona_2_f/r; (26) MERS (Betacoronavirus), primers gammacorona_f/r; (27) negative control for primers gammacorona_f/r; (29) TBEV, primers flavi_f/r; (30) YFV, primers flavi_f/r; (31) JEV, primers flavi_f/r; (32) negative control for primers flavi_f/r; (34) RABV, primers lyssa_f/r; (35) negative control for primers lyssa_f/r.

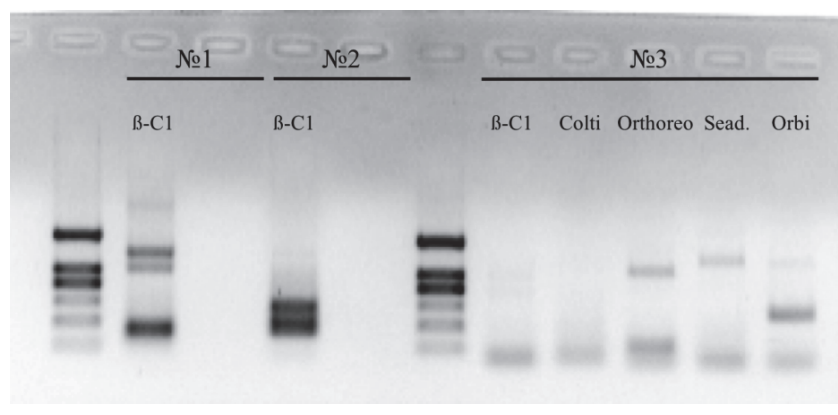

FIGURE 2: Reamplification of the multiplex PCR product; $B-C 1$ : Betacoronavirus-specific primers; Colti: coltivirus-specific primers; Orthoreo: Orthoreovirus-specific primers; Sead.: Seadornavirusspecific primers; Orbi: orbivirus-specific primers.

libraries preparation was performed using Ion Xpress Plus Fragment Library Kit (Thermo Fisher Scientific) according to the manufacturer's guide.

The quality of final libraries was assessed on the Agilent 2100 Bioanalyzer (Agilent Genomics), employing the Agilent High Sensitivity DNA Kit (Agilent Genomics).
Amplicon libraries were separated by $1.7 \%$ agarose gel electrophoresis stained with ethidium bromide, and the fragments of target lengths were cut out and purified using the MinElute Gel Extraction Kit (Qiagen). Size selection of the final total RNA libraries was done using $2 \%$ E$\mathrm{Gel}^{\mathrm{TM}}$ SizeSelect ${ }^{\mathrm{TM}}$ II Agarose Gels (Thermo Fisher Scientific) on the E-Gel electrophoresis system (Thermo Fisher Scientific).

Sequencing was carried out on the Ion S5 platform using Ion 520/530 Kit-Chef reagent sample preparation kit and employing Ion 530 chips on the Ion Chef instrument (Thermo Fisher Scientific).

2.9. Bioinformatics Analysis. Raw sequencing reads obtained from the platform were first filtered using the PRINSEQ-lite tool [23] to eliminate too short $(<80 \mathrm{bp})$ and low-quality (min_mean < 20) fragments. The mean, median, and 10th and 90th percentiles of read lengths distributions for selected unfiltered FASTQ files are shown in the supplementary Table S1. The BWA software [24] was used then to align the filtered reads to the reference birds' genomes database. The ideally aligned reads (full read length and no mismatches) were marked as nonviral reads and were eliminated. The software CD-HIT [21] was used to reduce the redundancy, i.e., the number of reads per sample, by clustering (with a similarity threshold 90\%) and selecting the representative sequences for each cluster. Briefly, the CD-HIT algorithm sorts the sequences from long to short and processes them sequentially. The first sequence is automatically classified as the first cluster representative sequence. Then each query sequence from the remaining sequences is compared to the representative sequences identified before it and is classified as redundant or representative based on whether it is similar to one of the existing representative sequences. The described filtering and clustering steps significantly reduced (by $\sim 100$ times) the computational time of the further steps. The software BLAST was then employed to first compare the representative sequences (RS) against virus-only nucleotide and protein databases that were collected by selection of virus sequences from GenBank NT and GenBank NR databases, respectively, to identify viral nucleotide candidate RS (nRS) and protein candidate RS (pRS) with E-value cutoffs of $10^{-5}$ and $10^{-3}$, respectively. Since virus-only databases are much smaller than NCBI NT and NR databases, pairwise sequence comparisons are much faster, which maximizes the speed and allows for the more efficient use of computational resources. We subsequently aligned these candidate nRS and $\mathrm{pRS}$ to the entire GenBank NT and NR databases, respectively, to eliminate potential false positives (sequences with higher similarity to nonviral reference sequences than to viral ones in the database) among the candidates and to select the true positives. The additional step was applied to select true positives from viral pRS: the pRS aligned to NR database were compared with nRS aligned to NT database to eliminate viral $\mathrm{pRS}$, which correspond to nRS aligning to nonviral nucleotide sequences with a high E-value. To get an idea of the total number of true viral reads belonging to the same virus, the numbers of reads for each RS were eventually summed up. 


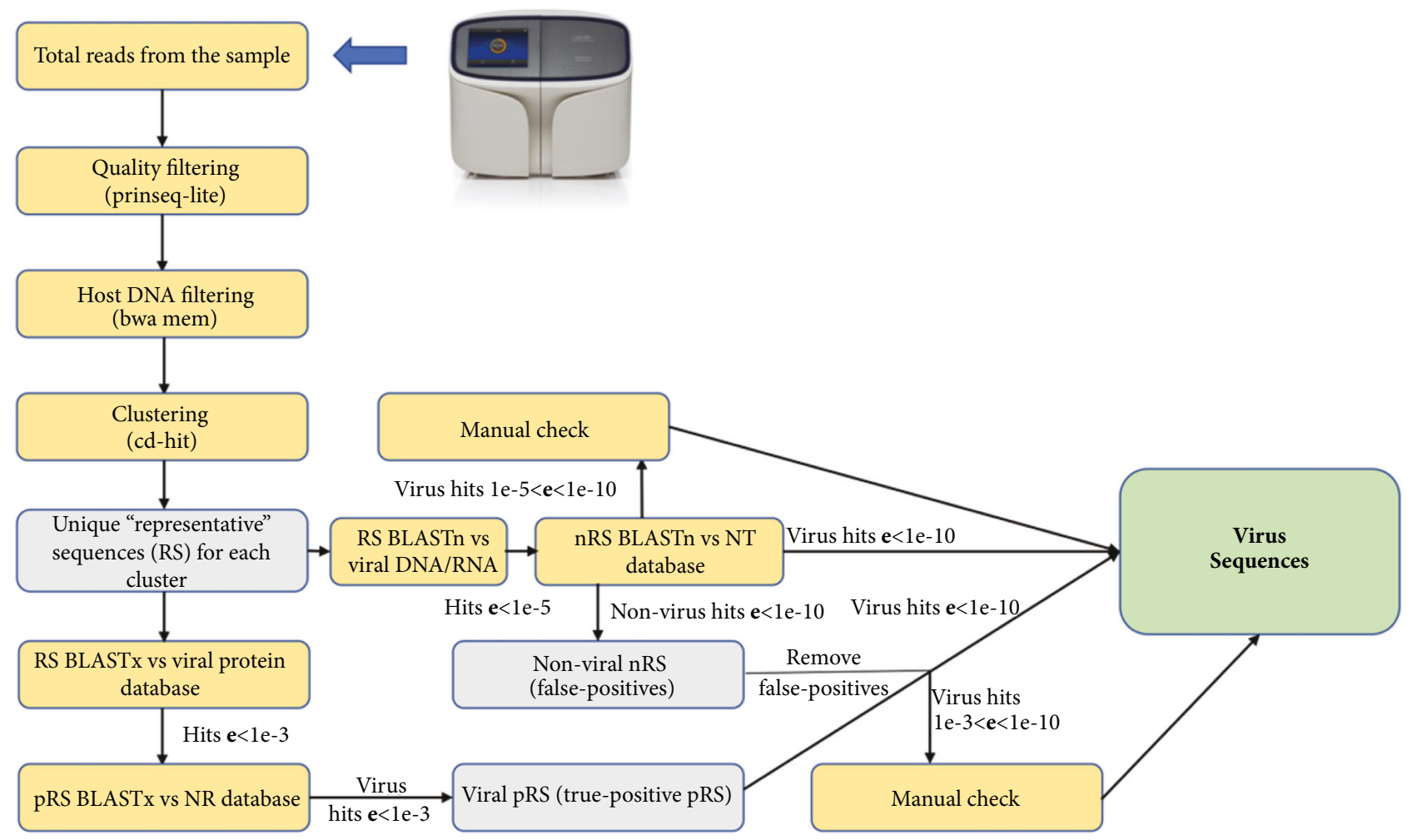

Figure 3: A schematic picture of the bioinformatics pipeline developed for the analysis of the NGS data in this study. Specific third-party tools that were employed are shown in parentheses.

A schematic picture of the bioinformatics analysis scheme is presented in Figure 3.

\section{Results and Discussion}

A panel of 45 primers including 23 forward and 22 reverse primers was designed using the developed algorithm and was synthesized accordingly (Tables 1 and 2). A preliminary analysis using the available control samples (Table 1) demonstrated the ability of the designed primers to perform targeted cDNA enrichment in both single-plex and multiplex formats. This was confirmed by the presence of specific bands of expected lengths in electrophoresis, followed by capillary sequencing (Figures 1 and 2).

Subsequently, we deployed the primer panel to study 70 bird samples (as described in the Methods) and the sequencing results are presented in Table 3 and Figure 4. Only those samples, for which at least 10 viral reads (filtered by quality and length) were identified in the final FASTQ files, are presented in the table. The total number of sequencing reads in such samples is also given to exemplify the percentage of pathogen reads. The alignment of sequencing reads with viral reference sequences was visually inspected to eliminate most of the potential artifacts (such as primer-dimers).

As evident from Table 3, the genera of viruses (samples $B 11, B 23$, and B24, highlighted in bold) having a specific primer pair in the panel showed significant amplification, and percentage of the corresponding viral reads ranged between $4.3 \%$ and $43.1 \%$. This confirms that our primer panel can efficiently enrich the target genera. Other sequencing reads belonged to bacteriophages, bacteria, and host species. However, we also observed a significant enrichment in the samples $B 27$ and $B 68$, for which a significant number of reads corresponding to Sanxia water strider virus and Duck adenovirus were, respectively, detected. We scrutinized these results in further detail by carrying out a BLAST analysis of all possible versions of the primers (since they are very degenerated) against the sequencing reads and observed that these findings were very likely due to nonspecific amplification from the primers designed for other genera (gammacoronal_f and flavi_r), though we have not checked this by a direct experiment with a panel that lacks these primers. Although nonspecific amplification allows an even more exhaustive search, it also introduces potentially undesirable enrichment. This could be a problem, especially when the lengths of obtained amplicons are outside the standard intervals suitable for sequencing on most popular platforms.

We also observed a considerable number of viral reads corresponding to various genera in some samples $(B 27, B 46$, $B 49, B 58, B 66$, and B69), despite the absence of specific primers in the panel for their enrichment. The number of reads varied from $0.00013 \%$ (Tunis virus) to $2.2 \%$ (Watercress white vein virus). The latter is a plant virus and its presence is expected as the feces of the host birds mainly contained grass. These results are possibly due to a weak, nonspecific PCR amplification. 
TABLE 3: Results of the bird samples sequencing with prior enrichment using the primers panel. The number of viral reads and total number of reads per corresponding sample are shown. For three samples (B11, B23, and B24, highlighted in bold) there was a specific primer pair in the panel present for the amplification of the detected genus. Closest viral homologs names and their GenBank IDs are shown.

\begin{tabular}{|c|c|c|c|c|c|}
\hline Bird ID & $\begin{array}{c}\text { Viruses (closest } \\
\text { homologs) detected }\end{array}$ & $\begin{array}{l}\text { Host } \\
\text { specie }\end{array}$ & $\begin{array}{l}\text { GenBank IDs of the } \\
\text { detected viruses }\end{array}$ & $\begin{array}{l}\text { Number of viral } \\
\text { reads in the } \\
\text { sample }\end{array}$ & $\begin{array}{l}\text { Total number of } \\
\text { reads obtained for } \\
\text { the sample }\end{array}$ \\
\hline$B 11$ & $\begin{array}{c}\text { Duck-dominant } \\
\text { coronavirus|Avian } \\
\text { coronavirus|Bird } \\
\text { droppings coronavirus }\end{array}$ & $\begin{array}{l}\text { Taiga Bean } \\
\text { Goose }\end{array}$ & $\begin{array}{c}\text { AKQ98474.1, } \\
\text { AKQ98475.1, APU51837.1, } \\
\text { AIY51827.1, CAH69463.1 }\end{array}$ & 4,342 & 108,833 \\
\hline$B 23$ & $\begin{array}{c}\text { Duck-dominant } \\
\text { coronavirus|Avian } \\
\text { coronavirus|Bird } \\
\text { droppings coronavirus } \\
\end{array}$ & $\begin{array}{l}\text { Taiga Bean } \\
\text { Goose }\end{array}$ & $\begin{array}{c}\text { AKQ98474.1, } \\
\text { AKQ98475.1, APU51837.1, } \\
\text { AIY51827.1, CAH69463.1 }\end{array}$ & 83,730 & 194,156 \\
\hline B24 & $\begin{array}{c}\text { Duck-dominant } \\
\text { coronavirus }\end{array}$ & $\begin{array}{c}\text { Taiga Bean } \\
\text { Goose }\end{array}$ & AKQ98474.1, AKQ98475.1 & 4,554 & 90,430 \\
\hline$B 27$ & $\begin{array}{c}\text { Sanxia water strider virus } \\
16 \\
\end{array}$ & $\begin{array}{c}\text { Temminck's } \\
\text { Stint }\end{array}$ & YP_009337377.1 & 4,062 & 103,488 \\
\hline$B 27$ & $\begin{array}{c}\text { Fowl aviadenovirus } \\
\text { C|Turkey aviadenovirus B }\end{array}$ & $\begin{array}{l}\text { Temminck's } \\
\text { Stint }\end{array}$ & $\begin{array}{c}\text { ACL68145.1, ANB27700.1, } \\
\text { ALY06332.1, ALY06333.1 }\end{array}$ & 54 & 103,488 \\
\hline$B 27$ & Cimodo virus & $\begin{array}{l}\text { Temminck's } \\
\text { Stint }\end{array}$ & YP_009059075.1 & 160 & 103,488 \\
\hline$B 46$ & $\begin{array}{c}\text { Watercress white vein virus } \\
\text { | Turnip yellow mosaic } \\
\text { virus }\end{array}$ & $\begin{array}{l}\text { Taiga Bean } \\
\text { Goose }\end{array}$ & AFC95826.1, AMH40128.1 & 636 & 28,468 \\
\hline B49 & Circovirus & $\begin{array}{c}\text { Taiga Bean } \\
\text { Goose }\end{array}$ & AEL87792.1 & 180 & 149,230 \\
\hline B58 & $\begin{array}{c}\text { Lake Sarah-associated } \\
\text { circular virus-32 }\end{array}$ & $\begin{array}{c}\text { Taiga Bean } \\
\text { Goose }\end{array}$ & ALE29729.1 & 10 & 42,348 \\
\hline$B 66$ & Duck aviadenovirus B & Fieldfare & YP_009047166.1 & 198 & 159,604 \\
\hline$B 68$ & Duck adenovirus A & Common tern & AGS11269.1, NP_044717.1 & 95,872 & 256,549 \\
\hline$B 69$ & Tunis virus & $\begin{array}{c}\text { Taiga Bean } \\
\text { Goose }\end{array}$ & AMT75434.1 & 16 & 126,648 \\
\hline
\end{tabular}

TABLE 4: Comparison of the sequencing results of two samples with and without preenrichment by the primer's panel. In the latter case, the total number of reads was $\sim 4.5$ million per sample.

\begin{tabular}{lcccc}
\hline & & B23 & & B66 \\
& With enrichment & Total RNA & With enrichment & Total RNA \\
\hline Coronaviruses & $43.1 \%$ & $0.2 \%$ & - & - \\
Adenoviruses & - & $0.2 \%$ & $0.1 \%$ & $0.05 \%$ \\
\hline
\end{tabular}

We then checked whether the obtained viral reads for samples B11, B23, B24, B27, and B68 were due to the amplification with the designed primers in the panel. These five samples were chosen as they indicated the most significant amplification, with $4.0-43.1 \%$ of total sequencing reads in the FASTQ files being viral. This was done by repeating a BLAST analysis of all possible versions of the primer sequences against the obtained viral reads for the aforementioned samples and calculating the percentage of those containing at least one primer. We observed that over $99 \%$ of the sequences contained the primers validating the effectiveness of the panel.

Finally, in order to confirm the effectiveness of the panel's enrichment, we selected two samples (B23 and B66) for which the presence of viral RNA was shown using amplicon sequencing data (see Table 3). Notably, the B23 possessed a significant number of reads that correspond to coronaviruses (43.1\%) and our panel had specific primers for these genera. As for the $B 66$ sample, it had a small number of reads that correspond to adenoviruses $(0.1 \%)$ but we did not design oligonucleotides for them. We then prepared total RNA-seq libraries for these samples, and subsequently sequenced them, yielding $\sim 4.5$ million of reads per sample. The percentage of the coronavirus reads in the $B 23$ sample was found to be significantly lower $(0.2 \%)$ than that in the same sample that was previously enriched by the panel (Table 4). As for the $B 66$ sample, we identified that $0.05 \%$ of reads belong to the Adenoviridae family, and most of them were identified as being similar to the Duck and Psittacine adenoviruses. This is similar to the percentage of reads found in the $B 66$ 

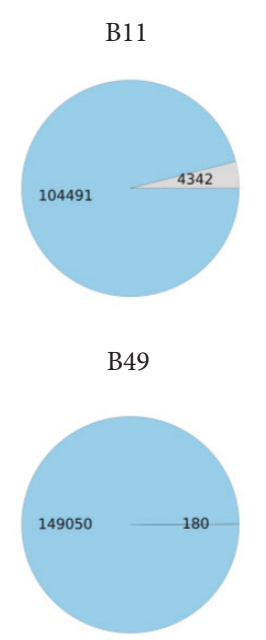

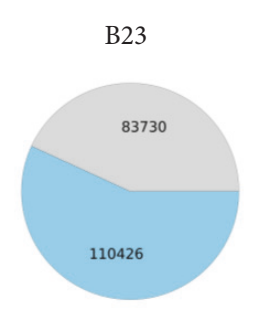

B58

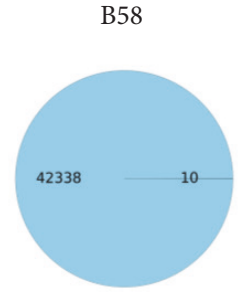

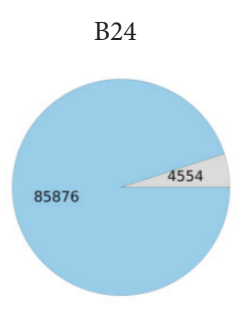

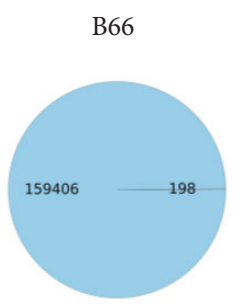

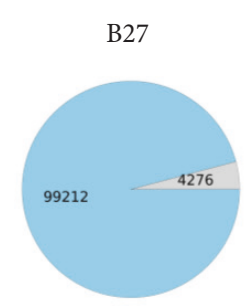

B68

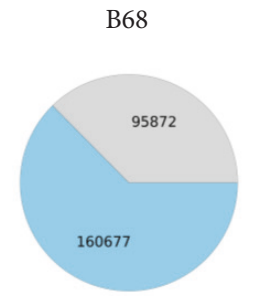

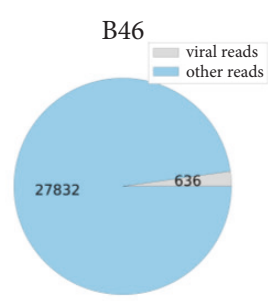

B69

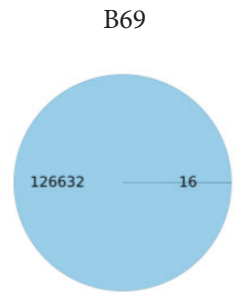

FIGURE 4: A graphical representation of the percentage of the detected viral reads (grey) with respect to the total number of sequencing reads obtained for the samples listed in Table 3.

FASTQ file (0.1\%) when the primer panel was applied, i.e., no noticeable enrichment was observed as expected. At the same time, we lost $\sim 0.2 \%$ of adenoviruses in the $B 23$, but since the amount of data was very different in the two experiments, there was a significant amplification of coronaviruses with almost a half of the reads belonging to these genera; this outcome is not totally surprising. Thus, our approach allows a significant reduction in the cost of sequencing. Besides, for a successful identification of viruses in a preenriched sample, $100,000-200,000$ reads per sample are generally enough.

\section{Conclusions}

In this study, we have presented a method of oligonucleotide design for the enrichment of viral nucleic acids. We used this method to design a primer panel, which showed a high efficiency in the detection and identification of several viruses using NGS sequencing. This is especially important for the detection of viruses known to persist in natural zoonotic reservoirs including birds, bats, and rodents, those with a high genetic diversity, and those which can be potentially dangerous to humans and animals. These factors make the development of rapid test-systems essential for the effective management of potential epidemics.

The described method can be recommended to the researchers investigating diverse viromes across different types of biological samples. We deployed our panel for the screening of a number of bird samples and demonstrated a great efficiency. In future studies, the panel will be expanded to target more diverse viruses and tested with bird samples from other regions.

\section{Data Availability}

All the FASTQ files used to support the findings of this study are available from the corresponding author upon request (Kamil Khafizov; kkhafizov@gmail.com).

\section{Ethical Approval}

All applicable international, national, and/or institutional guidelines for the care and use of animals were followed.

\section{Disclosure}

An earlier version of this work was presented as an abstract at The 43rd FEBS Congress Prague 7-12 July 2018.

\section{Conflicts of Interest}

The authors declare that there are no conflicts of interest regarding the publication of this paper.

\section{Authors' Contributions}

Andrey A. Ayginin, Vladimir G. Dedkov, German A. Shipulin, and Kamil Khafizov participated in research design, Ekaterina V. Pimkina, Alina D. Matsvay, Marina V. Safonova, Anna S. Speranskaya, Ekaterina A. Blinova, and Ilya V. Artyushin collected the samples and conducted experiments, Andrey A. Ayginin and Kamil Khafizov developed the algorithm and performed data analysis, Andrey A. Ayginin, Alina D. Matsvay, Marina V. Safonova, Vladimir G. Dedkov, German A. Shipulin, and Kamil Khafizov drafted the manuscript, and Andrey A. Ayginin, Ekaterina V. Pimkina, Alina D. Matsvay, Anna S. Speranskaya, Ekaterina A. Blinova, Marina V. Safonova, Ilya V. Artyushin, Vladimir G. Dedkov, German A. Shipulin, and Kamil Khafizov approved the final manuscript.

\section{Acknowledgments}

This work was supported by RSF Grant no. 17-74-20096. 


\section{Supplementary Materials}

Table S1: mean, median, and 10th and 90th percentiles of read lengths distributions for unfiltered FASTQ files. (Supplementary Materials)

\section{References}

[1] B. A. Jones, D. Grace, R. Kock et al., "Zoonosis emergence linked to agricultural intensification and environmental change," Proceedings of the National Acadamy of Sciences of the United States of America, vol. 110, no. 21, pp. 8399-8404, 2013.

[2] J. S. Mackenzie and M. Jeggo, "Reservoirs and vectors of emerging viruses," Current Opinion in Virology, vol. 3, no. 2, pp. 170-179, 2013.

[3] C. Wang, J. Wang, W. Su et al., "Relationship between domestic and wild birds in live poultry market and a novel human H7N9 virus in China," The Journal of Infectious Diseases, vol. 209, no. 1, pp. 34-37, 2014.

[4] V. A. Parvathy, V. P. Swetha, T. E. Sheeja, and B. Sasikumar, "Detection of plant-based adulterants in turmeric powder using DNA barcoding," Pharmaceutical Biology, vol. 53, no. 12, pp. 1774-1779, 2015.

[5] T. E. Berry, S. K. Osterrieder, D. C. Murray et al., "DNA metabarcoding for diet analysis and biodiversity: A case study using the endangered Australian sea lion (Neophoca cinerea)," Ecology and Evolution, vol. 7, no. 14, pp. 5435-5453, 2017.

[6] S. Jaenicke, C. Ander, T. Bekel et al., "Comparative and joint analysis of two metagenomic datasets from a biogas fermenter obtained by 454-pyrosequencing," PLoS ONE, vol. 6, no. 1, Article ID e14519, 2011.

[7] S. Günther and O. Lenz, "Lassa virus", Critical Reviews in Clinical Laboratory Sciences, vol. 41, no. 4, pp. 339-390, 2004.

[8] R. Schlaberg, C. Y. Chiu, S. Miller, G. W. Procop, and G. Weinstock, "Validation of metagenomic next-generation sequencing tests for universal pathogen detection," Archives of Pathology \& Laboratory Medicine, vol. 141, no. 6, pp. 776-786, 2017.

[9] T. Briese, A. Kapoor, N. Mishra et al., "Virome Capture Sequencing Enables Sensitive Viral Diagnosis and Comprehensive Virome Analysis," mBio, vol. 6, no. 5, 2015.

[10] D. R. VanDevanter et al., "Detection and analysis of diverse herpes-viral species by consensus primer PCR," Journal of Clinical Microbiology, vol. 34, no. 7, pp. 1666-1671, 1996.

[11] S. J. Anthony, J. H. Epstein, K. A. Murray et al., "A strategy to estimate unknown viral diversity in mammals," MBio, vol. 4, no. 5, Article ID e00598-13, 2013.

[12] S. Weiss, P. T. Witkowski, B. Auste et al., "Hantavirus in bat, Sierra Leone," Emerging Infectious Diseases, vol. 18, no. 1, pp. 159-161, 2012.

[13] C. Drosten et al., "Identification of a novel coronavirus in patients with severe acute respiratory syndrome," The New England Journal of Medicine, vol. 348, no. 20, pp. 1967-1976, 1967.

[14] L. Zheng, J. Tang, G. R. G. Clover, M. E. Spackman, A. J. Freeman, and B. C. Rodoni, "Novel genus-specific broad range primers for the detection of furoviruses, hordeiviruses and rymoviruses and their application in field surveys in South-east Australia," Journal of Virological Methods, vol. 214, pp. 1-9, 2015.

[15] S. K. Choi, J. K. Choi, W. M. Park, and K. H. Ryu, "RT-PCR detection and identification of three species of cucumoviruses with a genus-specific single pair of primers," Journal of Virological Methods, vol. 83, no. 1-2, pp. 67-73, 1999.
[16] M. Pfeffer, B. Proebster, R. M. Kinney, and O.-R. Kaaden, "Genus-specific detection of alphaviruses by a semi-nested reverse transcription-polymerase chain reaction," The American Journal of Tropical Medicine and Hygiene, vol. 57, no. 6, pp. 709$718,1997$.

[17] V. G. Dedkov, A. N. Lukashev, A. A. Deviatkin et al., "Retrospective diagnosis of two rabies cases in humans by high throughput sequencing," Journal of Clinical Virology, vol. 78, pp. 74-81, 2016.

[18] M. Y. Galperin, X. M. Fernández-Suárez, and D. J. Rigden, “The 24th annual Nucleic Acids Research database issue: a look back and upcoming changes," Nucleic Acids Research, vol. 45, no. 9, article 5627, 2017.

[19] B. E. Pickett, E. L. Sadat, Y. Zhang et al., "ViPR: an open bioinformatics database and analysis resource for virology research," Nucleic Acids Research, vol. 40, no. 1, pp. D593-D598, 2012.

[20] M. A. Larkin, G. Blackshields, N. P. Brown et al., "Clustal W and clustal X version 2.0," Bioinformatics, vol. 23, no. 21, pp. 29472948, 2007.

[21] W. Li and A. Godzik, "Cd-hit: a fast program for clustering and comparing large sets of protein or nucleotide sequences," Bioinformatics, vol. 22, no. 13, pp. 1658-1659, 2006.

[22] A. Untergasser, I. Cutcutache, T. Koressaar et al., "Primer3-new capabilities and interfaces," Nucleic Acids Research, vol. 40, no. 15, p. el15, 2012.

[23] R. Schmieder and R. Edwards, "Quality control and preprocessing of metagenomic datasets," Bioinformatics, vol. 27, no. 6, pp. 863-864, 2011.

[24] H. Li and R. Durbin, "Fast and accurate short read alignment with Burrows-Wheeler transform," Bioinformatics, vol. 25, no. 14, pp. 1754-1760, 2009. 


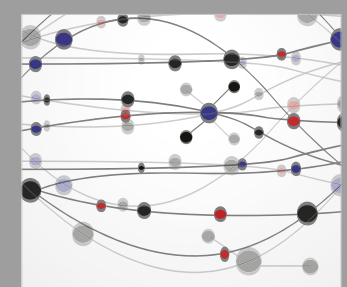

The Scientific World Journal
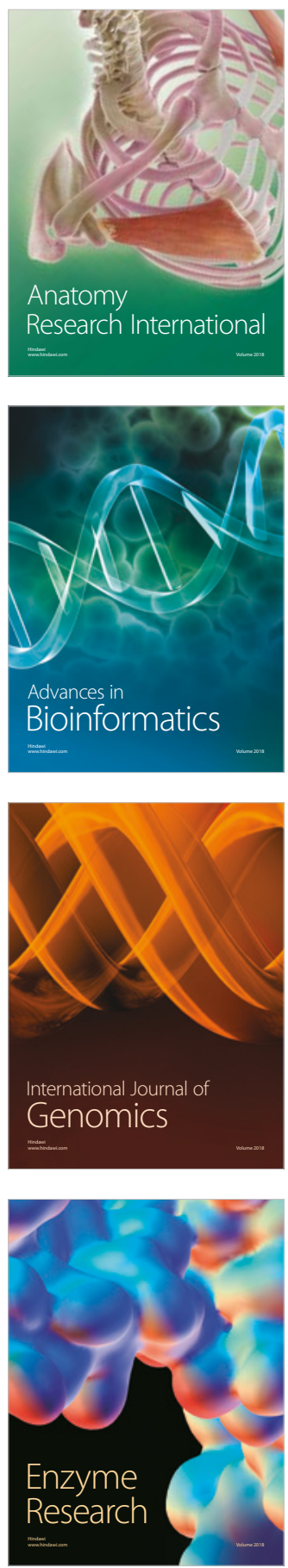
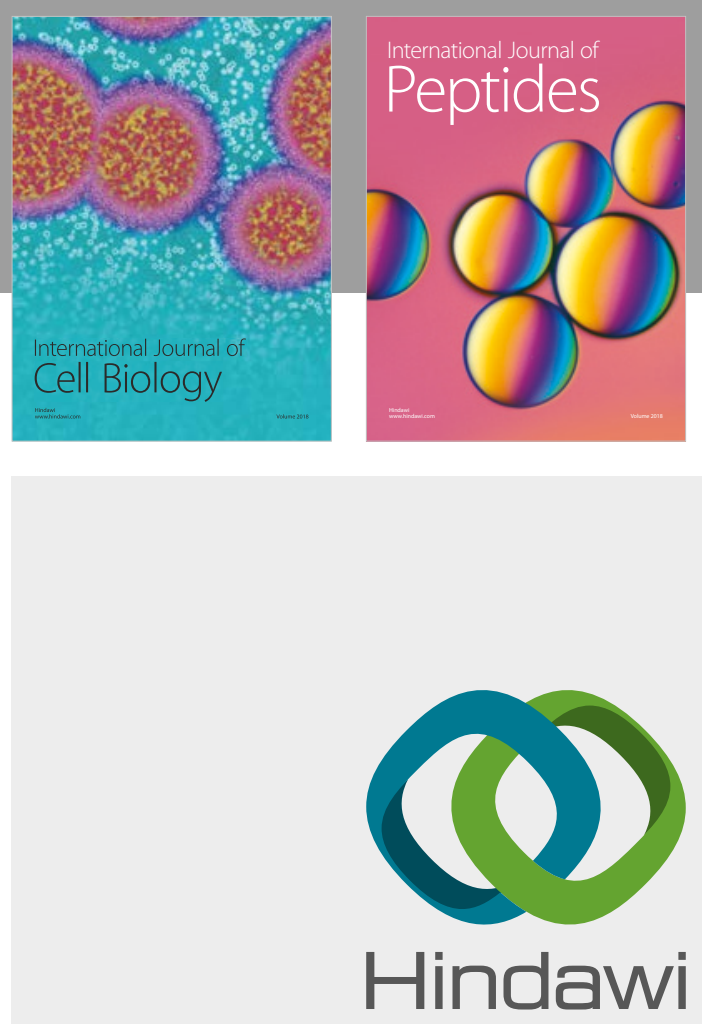

Submit your manuscripts at

www.hindawi.com
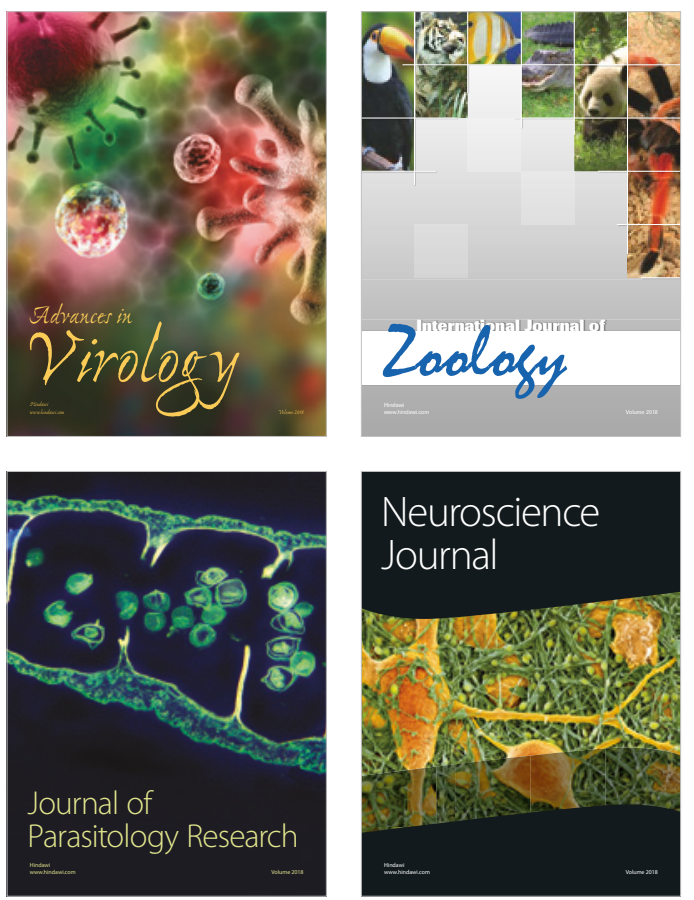
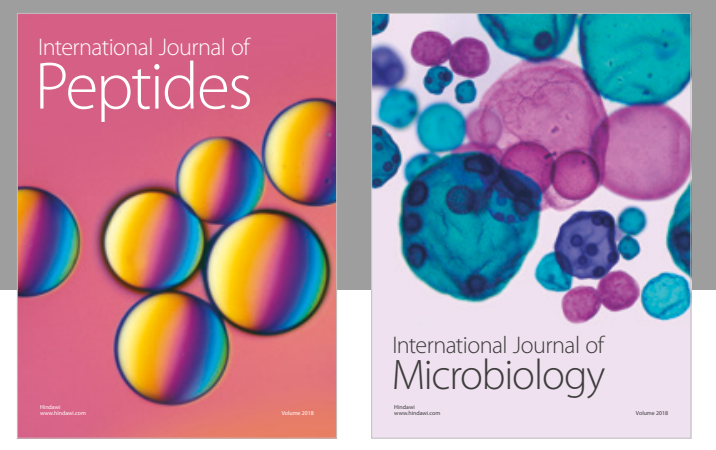

nternational Journal of Microbiology
Journal of
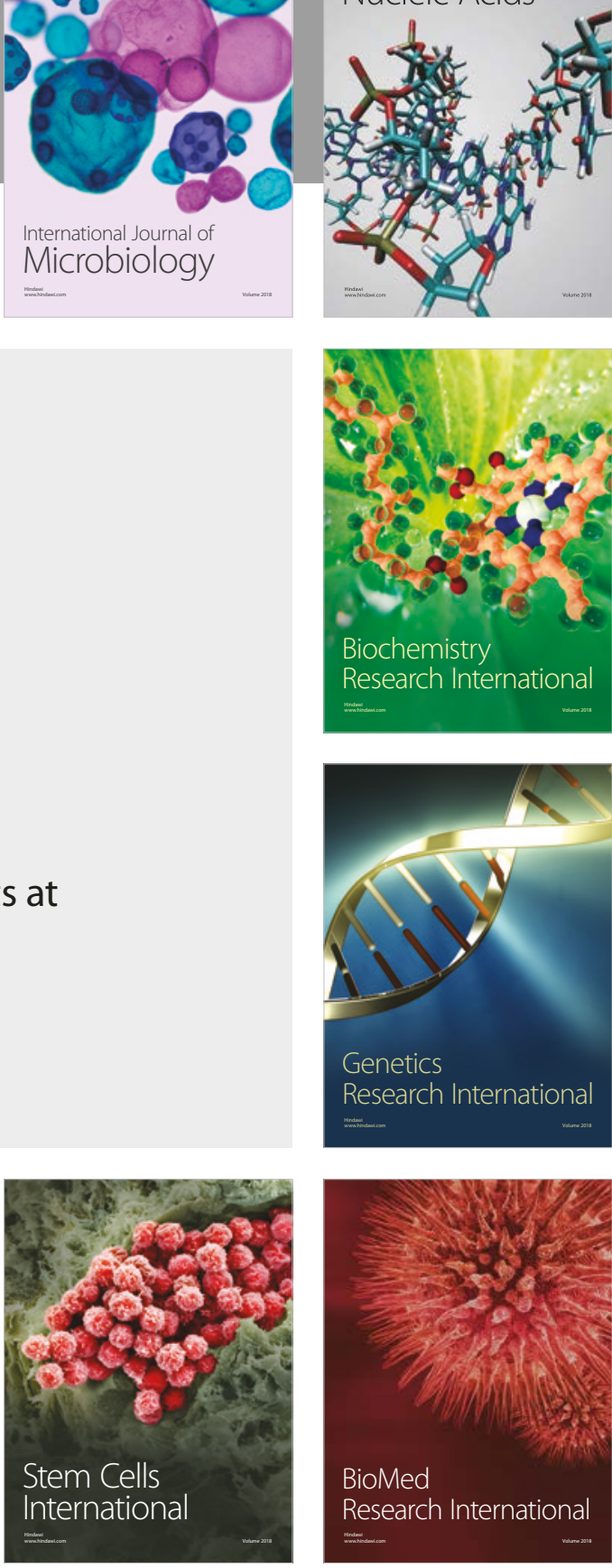
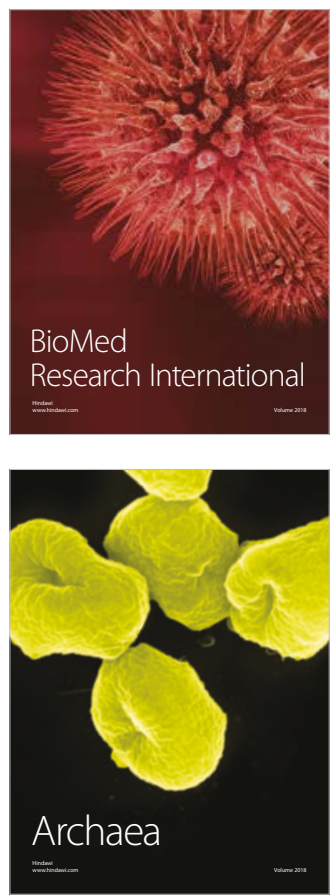